\title{
The Case for the Precision Timed (PRET) Machine
}

Stephen Edwards

Edward A. Lee

Electrical Engineering and Computer Sciences University of California at Berkeley

Technical Report No. UCB/EECS-2006-149

http://www.eecs.berkeley.edu/Pubs/TechRpts/2006/EECS-2006-149.html

November 17, 2006 
Copyright @ 2006, by the author(s).

All rights reserved.

Permission to make digital or hard copies of all or part of this work for personal or classroom use is granted without fee provided that copies are not made or distributed for profit or commercial advantage and that copies bear this notice and the full citation on the first page. To copy otherwise, to republish, to post on servers or to redistribute to lists, requires prior specific permission.

\section{Acknowledgement}

Edwards is supported by NSF, Intel, Altera, the SRC, and NYSTAR. Lee received support this work from NSF award number CNS-0647591 and from the Center for Hybrid and Embedded Software Systems (CHESS) at UC Berkeley, which receives support from the National Science Foundation (NSF award CCR-0225610), the State of California Micro Program, and the following companies: Agilent, DGIST, General Motors, Hewlett Packard, Infineon, Microsoft, National Instruments, and Toyota. 


\title{
The Case for the Precision Timed (PRET) Machine
}

\author{
Stephen A. Edwards* \\ Edward A. Lee ${ }^{\dagger}$
}

November 17, 2006

\begin{abstract}
We argue that at least for embedded software applications, computer architecture, software, and networking have gone too far down the path of emphasizing average case performance over timing predictability. In architecture, techniques such as multi-level caches and deep pipelines with dynamic dispatch and speculative execution make worst-case execution times (WCET) highly dependent on both implementation details of the processor and on the context in which the software is executed. Yet virtually all real-time programming methodologies depend on WCET. When timing properties are important in the software and when concurrent execution is affected by timing, the result is brittle designs. In this paper, we argue for precision timed (PRET) machines, which deliver high performance, but not at the expense of timing predictability. We summarize a number of research approaches that can be used to create PRET machines, and discuss how the software, operating system, and networking abstractions built above the machine architecture will have to change.
\end{abstract}

\section{The Problem}

Patterson and Ditzel [12] did not invent reduced instruction set computers (RISC) in 1980. Earlier computers all had reduced instruction sets. Instead, they argued that trends in computer architecture had gotten off the sweet spot, and that by dropping back a few years and forking a new version of architectures, leveraging what had been learned, they could get better computers by employing simpler instruction sets.

It is again time for a change in direction in computer architecture. Architectures currently strive for superior average-case performance that regrettably ignores predictability and repeatability of timing properties. "Correct" execution of the SPECint

\footnotetext{
* Edwards is with Columbia University and is supported by NSF, Intel, Altera, the SRC, and NYSTAR.

${ }^{\dagger}$ Lee is with UC Berkeley and received support this work from NSF award number CNS-0647591 and from the Center for Hybrid and Embedded Software Systems (CHESS) at UC Berkeley, which receives support from the National Science Foundation (NSF award CCR-0225610), the State of California Micro Program, and the following companies: Agilent, DGIST, General Motors, Hewlett Packard, Infineon, Microsoft, National Instruments, and Toyota.
} 
benchmark suite has nothing to do with how long it takes to perform any particular action. C says nothing about timing, so timing is not considered part of correctness. Architectures have developed deep pipelines with speculative execution and dynamic dispatch. Memory architectures have developed multi-level caches and TLBs. The performance criterion is simple: faster (on average) is better.

The biggest consequences have been in embedded computing. Avionics offers an extreme example: in "fly by wire" aircraft, where software interprets pilot commands and transports them to actuators through networks, certification of the software is extremely expensive. Regrettably, it is not the software that is certified but the entire system. If a manufacturer expects to produce a plane for 50 years, it needs a 50-year stockpile of fly-by-wire components that are all made from the same mask set on the same production line. Even a slight change or "improvement" might affect timing and require the software to be re-certified.

Nearly every abstraction provided by computing has failed our poor aircraft manufacturer. The instruction-set architecture, meant to hide hardware implementation details from the software, has failed because the user of the ISA cares about timing properties that the ISA does not guarantee. The programming language, which hides details of the ISA from the program logic, has failed because no widely used programming language expresses timing properties. Timing is merely an accident of the implementation. A real-time operating system hides details of the programs from the concurrent orchestration, yet this fails because the timing may affect the orchestration. The RTOS provides no guarantees. The network hides details of electrical or optical signaling from systems, but standard networks provide no timing guarantees, and hence again fail to provide an appropriate abstraction. The aircraft manufacturer is stuck with a system design (not just implementation) in silicon and wires.

All embedded systems designers face less extreme versions of this problem. "Upgrading" a microprocessor in an engine control unit for a car requires thorough retesting of the system. Even "bug fixes" in the software can be extremely risky, since they can change timing behavior and produce effects that were never seen in testing.

Even general-purpose computing suffers from these decisions. Since timing is neither specified in programs nor enforced by execution platforms, a program's timing properties are not repeatable. Buggy concurrent software often has timing-dependent behavior; small changes in the timing of one part of a program can affect seemingly unrelated parts.

Designers traditionally covered these failures by computing worst case execution time (WCET) bounds and using real-time operating systems (RTOSes) with predictable scheduling policies. But these require substantial margins for reliability, and ultimately reliability is (weakly) determined by bench testing of the complete implementation.

Modern processor architectures render WCET virtually unknowable; even simple problems demand heroic efforts. For example, Ferdinand et al. [5] determine the WCET of astonishingly simple avionics code from Airbus running on a Motorola ColdFire 5307, a pipelined CPU with a unified code and data cache. Despite the software consisting of a fixed set of non-interacting tasks containing only simple control structures, their solution required detailed modeling of the seven-stage pipeline and its precise interaction with the cache, generating a large integer linear programming problem. The technique successfully computes WCET, but only with many caveats that are in- 
creasingly rare in software. Fundamentally, the ISA of the processor has failed to provide an adequate abstraction.

Timing behavior in RTOSes is coarse and becomes increasingly uncontrollable as the complexity of the system increases, e.g., by adding inter-process communication. Locks, priority inversion, interrupts and similar issues break the formalisms, forcing designers to rely on bench testing, which is nearly impotent at flushing out subtle timing bugs. Worse, these techniques produce brittle systems in which small changes can cause big failures.

Synchronous digital hardware - the technology on which most computers are builtcan deliver astonishingly precise, repeatable timing behavior, thanks in part to considerable efforts on the part of hardware designers and design tool builders. Software abstractions, however, lose several orders of magnitude in timing precision. Consider the nanosecond-scale precision with which hardware can raise an interrupt request to the imprecision with which a user-level software thread sees the effects (perhaps milliseconds).

Commercial RTOSes market predictable timing, but modern processors have rendered such numbers only vague bounds. Real-time software developers have long demanded predictable timing; processor architectures no longer deliver.

\section{The Solution}

It is time for a new era of processors whose temporal behavior is as easily controlled as their logical function. We call them precision timed (PRET) machines. Our basic argument is that real-time systems, in which temporal behavior is as important as logical function, are an important and growing application; processor architecture needs to follow suit.

This is an enormous problem, but it is easy to start making progress. The problem is challenging because it spans nearly all abstraction layers in computing, including programming languages, virtual memory, memory hierarchy, pipelining techniques, power management, I/O, DRAM design, bus architectures, memory management, justin-time (JIT) compilation, multitasking (threads and processes), task scheduling, software component technologies, and networking.

Our first step is to develop FPGA-targeted PRET cores suitable for high-reliability embedded applications. Substantial progress can be made in months; the revolution may take decades. Our ultimate goal is networked real-time software that delivers the reliability and timing precision of synchronous digital hardware with the simplicity of software.

Timing precision is easy to achieve if you are willing to forgo performance; the engineering challenge in PRET machines is to deliver both. While we cannot abandon structures such as caches and pipelines and 40 years of progress in programming languages, compilers, operating systems, and networking, many will have to be rethought.

Fortunately, there is much work on which to build. ISAs can be extended with instructions that deliver precise timing with low overhead [7]. Scratchpad memories can be used in place of caches [1]. Deep pipelines with pipeline interleaving can de- 
liver precise timing [10]. Memory management pause times can be bounded [2]. Programming languages can be extended with timed semantics [6]. Appropriately chosen concurrency models can be tamed with static analysis [3]. Software components can be made intrinsically concurrent and timed [11]. Networks can provide high-precision time synchronization [8]. Schedulability analysis can provide admission control, delivering run-time adaptability without timing imprecision [4].

Our vision of a mature PRET machine incorporates most of these techniques. At the ISA level, it provides cycle-accurate timers, a predictable memory hierarchy based on scratchpad memories, and an interleaved pipeline that provides predictable hardware-efficient concurrency. It will be programmed in a C-like language that includes user-specified timing constraints and concurrency, perhaps with synchronous semantics. Both compile- and run-time checks will ensure the program meets timing constraints, similar to array bounds checking. A PRET operating system will resemble an RTOS, but its scheduling policies will provide guarantees and admission control. Such a processor will communicate through a network able to provide timing guarantees, probably leveraging time synchronization.

Many open challenges remain. How do we achieve high-precision I/O (classical interrupts destroy all temporal predictability)? How do we manage disk systems, DRAM behavior, and virtual memory? How do we scale to deep sub micron without losing the precision of synchronous digital logic (see http://www.tauworkshop.com)? How do we adapt operating systems to provide timing guarantees? How do we handle exceptions? How do we handle variable clock rates (essential power management)? How do we get precise timing in networking? How do we evolve the many fledgling research results into mainstream software engineering?

PRET machines are essential for embedded systems, but are also valuable for general-purpose systems. In concurrent software, non-repeatable behavior is a major obstacle to reliability [9]. PRET machines would improve reliability of concurrent software through repeatable concurrent behavior.

Patterson and Ditzel's [12] plea for RISC machines was simultaneously heeded and ignored. Architectural complexity continued to grow unabated, but at least architects began to analyze where it would have the most benefit. It forced architects to evaluate the benefits of their elaborations relative to the costs. A similar change is needed with respect to techniques that blithely ignore predictable timing.

\section{References}

[1] O. Avissar, R. Barua, and D. Stewart. An optimal memory allocation scheme for scratch-pad-based embedded systems. Trans. Embedded Computing Sys., 1(1):6-26, 2002.

[2] D. F. Bacon, P. Cheng, and V. Rajan. The Metronome: A simpler approach to garbage collection in real-time systems. In Workshop Java Tech. for Real-Time and Embedded Sys., Catania, Sicily, 2003.

[3] G. Berry. The effectiveness of synchronous languages for the development of safety-critical systems. White paper, Esterel Tech., 2003.

[4] E. Bini and G. C. Buttazzo. Schedulability analysis of periodic fixed priority systems. IEEE Trans. Computers, 53(11):1462-1473, 2004.

[5] C. Ferdinand et al. Reliable and precise WCET determination for a real-life processor. In Proc. Conf. Embedded Software, volume 2211 of LNCS, pages 469-485, North Lake Tahoe, California, Oct. 2001. 
[6] T. A. Henzinger, B. Horowitz, and C. M. Kirsch. Giotto: A time-triggered language for embedded programming. In EMSOFT 2001, volume LNCS 2211, Tahoe City, CA, 2001. Springer-Verlag.

[7] N. J. H. Ip and S. A. Edwards. A processor extension for cycle-accurate real-time software. In Embedded and Ubiquitous Computing, volume 4096 of LNCS, pages 449-458, Seoul, Korea, Aug. 2006.

[8] S. Johannessen. Time synchronization in a local area network. IEEE Control Systems Magazine, pages 61-69, 2004.

[9] E. A. Lee. The problem with threads. Computer, 39(5):33-42, 2006.

[10] E. A. Lee and D. G. Messerschmitt. Pipeline interleaved programmable dsps: Architecture. IEEE Trans. on Acoustics, Speech, and Signal Processing, ASSP-35(9), 1987.

[11] E. A. Lee, S. Neuendorffer, and M. J. Wirthlin. Actor-oriented design of embedded hardware and software systems. Journal of Circuits, Systems, and Computers, 12(3):231-260, 2003.

[12] D. A. Patterson and D. R. Ditzel. The case for the reduced instruction set computer. ACM SIGARCH Computer Architecture News, 8(6):25-33, Oct. 1980. 